\title{
On connecting connected discourse: A comment on methodology*
}

\author{
RAYMOND W. KULHAVY, JAMES W. DYER $\dagger$, and LINDA C. CATERINO \\ Arizona State University, Tempe, Arizona 85281
}

\begin{abstract}
College subjects learned three 67-word narrative paragraphs under either serial or intact presentation modes. Subjects were also instructed to read for verbatim or substance content. Control subjects received no instructions. Leaners receiving intact presentation recalled significantly more of the paragraphs read. In addition, an analysis of words recalled in order indicated that subjects are able to employ a strategy set only if the discourse is presented as an intact unit. The data suggest that learning discourse in a serial manner may yield results quite different from those obtained with intact presentation.
\end{abstract}

The focus of this article is primarily methodological. Essentially, we believe that many studies using connected discourse save failed to take into account important aspects of what happens when a person learns contextually related material. Specifically, we are referring to studies which have presented passages in serial fashion. There is some evidence that the learning of discrete word lists is relatively uninfluenced by the type of presentation (Kulhavy \& Heinen, 1974). However, since both context relations and the control of scanning activities seem to be major variables for prose learning (e.g., Bransford \& Johnson, 1972; Heinen, Kulhavy, \& Dyer, 1974; Kulhavy, 1972), it is unclear why one would present meaningful discourse as single words. Control over exposure rates is a poor reason for employing the list procedure, since it is obvious that different words carry varying amounts of information-especially within the setting of an entire passage. If one is interested in what happens when people learn connected discourse, it seems odd that they would control for factors which appear essential for making discourse "connected."

In a cursory survey of the experimental literature from 1960 to 1973 , we were able to find over 20 studies in which connected material was presented by methods ranging from serial anticipation to flipping computer cards at a timed rate. Also, in cases where we were familiar with related research using intact presentation, there were often marked differences in both the results obtained and their interpretation. This information observation buttresses our contention that serial presentation may mask the every effects one hopes to obtain from their experiment.

In order to push our argument onto firmer empirical ground, we designed the simple study that follows. If, as we assume, intact exposure of connected discourse increases the amount and type of information available

*The authors wish to thank Anita Favela and Alta May Miller for their assistance in producing this study.

+ Now at the University of Montana, Missoula, Montana. to subject, then a total presentation condition should facilitate remember over that of its serial counterpart. Consequently, we predicted that subjects receiving entire passages should outperform those who saw the material one word at a time. Recall superiority for the intact passages should occur mainly because context relations are available for storage and subject's passage scanning behaviors are allowed a free idiosyncratic rein.

Finally, we reasoned that telling an individual to learn either verbatim content or substantive "gist" should have little effect on serial mode performance, simply because viewing each word separately constrains subject from applying other than sequential encoding strategies. On the other hand, when a learner sees the intact passage, it seems more likely that he will be able to conform to instructional task requirements because he has the entire contextual unit available to select from.

\section{METHOD}

\section{Design and Subjects}

The design was a 2 by 3 factorial with two levels of presentation mode (serial) vs total) and three levels of preexperimental instructions (substance vs verbatim vs read-only).

The subjects were 95 undergraduate volunteers attending Arizona State University. Subjects were randomly assigned, 15 to each factorial cell, in order of their appearance for the experiment. Five subjects were dropped from the study for failure to follow procedural instructions.

\section{Materials and Procedures}

The authors constructed three 67-word paragraphs titled: The Dump, The Forest, and The Traveler. Each of the paragraphs consisted of a self-contained descriptive narrative. The number of both content $(M=16.66)$ and function $(M=50.15)$ words was essentially the same for all three paragraphs. Verbal data from pilot subjects indicated that the passages were "easy" to read and readily comprehendable by a college population.

For the total presentation groups, each paragraph was typed double-spaced on a sheet of white foolscap. Words for the serial presentation were typed individually in the upper left corner of a white computer card. Punctuation characters were included on the card containing the word preceding them. The cards were presented in a small tray which allowed subject to turn them 
individually from one compartment to another. A blue card signaled the demarcation between each paragraph. The recall sheets consisted of 8-1/2 $\times 11$ in. pages of lined paper.

From one to three subjects participated in each experimental session. Subjects entered the experimental chamber, seated themselves across a table from experimenter, and heard standard instructions appropriate to their condition. Subjects in the verbatim condition were told to read for exact content, and to try and learn the paragraphs as close to their original words as possible. For the substance groups, learners were asked to read the paragraphs for substantive meaning and general "ideas." Finally, the controls were told simply to study the material carefully. All subjects were told that they would be tested following the learning session. In all cases, the order of paragraph presentation was counterbalanced so that each paragraph occurred in all positions and an equal number of times across conditions.

During the learning session, subjects in the serial condition turned the cards individually at a $1.5-\mathrm{sec}$ rate. Exposure time was controlled by beeps from a tape recorder. Subjects in the total condition were allowed to study the entire paragraph for $100 \mathrm{sec}$.

In order to preclude recall from STM, subject was required to work on a page of complex math problems for 1 min following completion of a passage. Immediately after the interpolated task, subject was handed a lined sheet and given $3 \mathrm{~min}$ to recall as much of the passage as he could remember. The entire study-test procedure was replicated exactly for each of the three paragraphs.

\section{RESULTS AND DISCUSSION}

The protocols were first scored for the total number of words recalled, discounting both intrusions and repetitions. Since there were no differences between the paragraphs in the amount recalled $(F=2.22)$, the data were pooled for the main analysis. A 3 (I) by 2 (P) analysis of variance on total recall yielded significance only for the $\mathrm{P}$ main effect, $\mathrm{F}(1,84)=17.67, \mathrm{p}<.001$. The mean words recalled were $116(\mathrm{SD}=31.50)$ and $140(\mathrm{SD}=23.76)$ for the serial and total conditions, respectively.

Obviously, if one accepts total recall as a gross measure of what is stored, the total group was able to remember substantially more of the material read. These are exactly the effects predicted by the introductory argument. The lack of results for the instructions factor was contrary to our expectations. Perhaps a measure that discounts sequence effects is less sensitive to the type of strategy employed by a subject. In any event, the total words analysis provides dramatic support for the contention that more is learned from intact presentation.

In order to assess how well verbatim sequences were remembered, a second analysis was done on words recalled in order. For this scoring, words were counted as correct if (a) there were at least three together in the original order and $(b)$ the group contained at least one content word. Again, both inttusions and repetitons were discounted in the scoring. A preliminary analysis comparing the three paragraphs was significant, $F(2,267)$ $=6.25, \mathrm{p}<.01$. Inspection of the recall means showed
Table 1

Means and Standard Deviations for Words Recalled in Order

\begin{tabular}{clccc}
\hline \multirow{2}{*}{$\begin{array}{c}\text { Presentation } \\
\text { Mode }\end{array}$} & & \multicolumn{3}{c}{ Instructions } \\
\cline { 3 - 5 } Serial & M & 23.67 & 29.13 & 25.53 \\
& SD & 16.45 & 19.14 & 15.90 \\
\multirow{2}{*}{ Total } & M & 69.13 & 45.53 & 63.13 \\
& SD & 27.17 & 21.10 & 19.73 \\
\hline
\end{tabular}

Note-Each mean is based on the recall of 15 subjects.

that more words in order were recalled from the Forest passage. We could find no explanation for this effect since it failed to occur in the total words analysis, and there were no obvious differences between the paragraphs in terms of length and structure. Consequently, because there appeared to be no interaction with either of the experimental factors, the paragraph scores were again pooled for the main analysis. The 3 (I) by 2 (P) ANOVA on ordered words showed significant effects for the $\mathrm{P}$ factor, $\mathrm{F}(1,84)=$ $62.16, p<.001$, and the $I$ by $P$ interaction, $F(2,84)=$ $5.18, \mathrm{p}<.01$. A comparison of simple effects across the I by $\mathbf{P}$ means showed that the differences were primarily restricted to the total condition, with the I means ranking in the order verbatim $=$ control $>$ substance $(\mathrm{p}<.01)$. The means and standard deviations for the words-in-order data are presented in Table 1.

As with the first analysis, the total groups markedly outperformed the serial conditions. This fact is not surprising, since both total and in-order recall share a large amount of variability $(r=.83)$. However, the performance decrement exhibited by subjects receiving substance instructions in the total condition indicates that subject can, and does, control how he encodes the passage material. Apparently a gist strategy, which reduces the amount of sequential recall can only be effectively employed if the passage is read.as a whole. These results demonstrate an important point. How discourse is presented can yield differential effects across levels of a contrasted variable-even with a vague manipulation such as verbal instructions. Quite possibly such differences would be even more pronounced if one was using a context-related measure such as "idea" units or theme (Bransford \& Johnson, 1972; Dooling \& Lachman, 1971).

In summary, our original point appears to be well made. The manner in which connected discourse is presented can determine the direction of one's experimental outcomes. If variables are viewed in terms of their relation to connected discourse, then the discourse should be connected during viewing.

\section{REFERENCES}

Bransford, J. D. \& Johnson, M. K. Contextual prerequisites for understanding: Some investigations of comprehension and 
recall. Journal of Verbal Learning and Verbal Behavior, 1972, 11, 717-726.

Dooling, D. J., \& Lachman, R. Effects of comprehenson on retention of prose. Journal of Experimental Psychology, retention of prose.

Heinen, J. R. K., Kulhavy, R. W., \& Dyer, J. W. Context and the meaning of words in paragraphs. Paper presented at the Annual Convention of the American Psychological
Association, New Orleans, August 1974.

Kulhavy $R$ W. Effects of embedding orienting stimuli in a prose passage. Psychonomic Science, 1972, 28, 213-214.

Kulhavy, R. W., \& Heinen, J. R. K. Mnemonic transformations and verbal coding processes. Journal of Experimental Psychology, 1974, 102, 173-175.

(Received for publication August 13, 1974.)

\title{
On the recall of nonverbal experiences
}

\author{
JOHN G. SEAMON \\ Wesleyan University, Middletown, Connecticut 06457
}

\begin{abstract}
The inability to recall early childhood experiences had led some theorists to suggest that the development of a visual representational system precedes that of a verbal system. Events perceived prior to the onset of the verbal system are unable to be recalled subsequently through that system. The present experiment, however, has demonstrated that subjects could attach verbal labels to nonverbal stimuli which were experienced prior to the establishment of rules for labeling. This suggests that poor recall of early experiences may reflect true forgetting rather than information inaccessibility. Assimilation into a permanent memory structure may be a necessary conditon for long-term retention.
\end{abstract}

Why are we unable to recall many events from our early childhood especially events which occurred before the age of two or three? A number of theorists who have considered this issue have suggested that there may be separate modes of thought which develop differentially in man. The two modes may be conceptualized as visual and verbal representational systems with the temporal onset of the former preceding that of the latter (Bower, 1970; Bruner, 1966; Gazzaniga, 1972; Neisser, 1962). Gazzaniga (1972, p. 315) summarizes this position of information inaccessibility by stating that "It is possible that the brain can remember critical events which may later play a role in the control of behavior, but because the remembered events occurred prior to the clear establishment of the language system they cannot be subsequently recalled through this system."

The present experiment was performed to examine an issue raised by the above theorists, viz, could the subjects attach verbal labels to nonverbal stimuli which were experienced prior to the establishement of rules for labeling? The ability of subjects to attach labels to their earlier nonverbal experiences would imply that our poor recall of early events may not be explained as a problem of information inaccessibility.

\section{METHOD}

Subjects

Thirty-two male and female Wesleyan University students

This research was supported by a Wealey an Univernity Faculty Research Grant. Requests for reprints should be sent to John G. Seamon, Department of Psychology, Wesleyan University, Middletown, Connecticut 06457.
}

served as paid subjects in this experiment.

\section{Materials}

The stimuli consisted of a pool of 18 nonsense shapes and 18 nonsense verbal labels individually printed on $7.5 \times 12.8 \mathrm{~cm}$ white cards. The shapes were geometric line drawings which varied in number of sides from 8 to 16 with a mean of 10.43 and filled the entire card. They were constructed by using only straight lines and forming angles by using a table of random numbers. The shapes generated were different from each other and not similar to any known geometric shapes or to any two-dimensional representations of real-life objects.

The nonsense labels, printed on separate cards, were pronounceable CVC syllables with no obvious meaningful associates e.g., RUP, DAK. The labels were typed in capital letters in the center of each card.

\section{Procedure}

The subjects were assigned to one of four experimental groups on a random basis and run individually. The groups differed by the order of presentation of the shape sequence and paired-associates (P-A) task, and by the type of stimuli used in the final recognition task. In the first condition (Shapes, P-A, 1) a subset of eight shapes was randomly selected for each subject from the pool of 18 and individually presented to the subjects with each card shown for $15 \mathrm{sec}$. The subjects were told to simply observe each shape and that additional instructions would follow. After the last shape was presented, a P-A task was employed in which the subjects had to learn a CVC label to each of eight shapes. The shapes used in the P-A task consisted of four shapes randomly selected from the previously viewed eight, and four completely new shapes not seen before and randomly selected from those remaining in the original pool. The subjects were not informed that some of the shapes in the P-A task were from the preceding sequence. A standard P-A format was used 